\title{
CyPass ${ }^{\circledR}$ Micro-Stent Safety and Efficacy at One Year: What Have We Learned?
}

\author{
Itay E Gabbay ${ }^{1}$, Simon Ruben ${ }^{2}$
}

\begin{abstract}
Purpose: The suprachoroidal pathway can potentially provide a viable target for intraocular pressure (IOP) control. We present efficacy and safety result for the CyPass ${ }^{\circledR}$ Micro-Stent that provides insight into the use of the suprachoroidal space for IOP reduction.

Materials and methods: A retrospective, noncomparative audit of patient records in a university-affiliated National Health Service (NHS) hospital between June 2017 and August 2018. Main outcome measures were IOP reduction and the number of glaucoma drops taken at each time point. Failure and adverse events were noted for each patient.

Results: Seventy-seven consecutive case records of 69 patients underwent the CyPass ${ }^{\circledR}$ procedure between August 2017 and August 2018 . A stand-alone procedure was performed in $37(58 \%)$ of cases and combined phacoemulsification and CyPass ${ }^{\circledR}$ procedures were performed in $27(42 \%)$ of cases. At baseline, the mean IOP was $24.6 \pm 7.2 \mathrm{~mm} \mathrm{Hg}$. The mean IOP at 12 months' follow-up was $16.4 \pm 4.5 \mathrm{~mm} \mathrm{Hg}(p<0.05)$. At baseline, the mean number of medications was $2.41 \pm 0.98$, which decreased at 12 months to $1.1 \pm 1.3(p<0.05)$. Best corrected visual acuity analysis did not show statistically significant change preoperatively and at each postoperative time point. At 12 months' postoperative time point, $24 \%$ of eyes had failed. Significant IOP spikes were noted in $18(28.1 \%)$ of cases.

Conclusion: The suprachoroidal pathway appears to potentially offer an alternative to the classic subconjunctival pathways, yet present a new set of challenges. Further studies would have to address these concerns of endothelial cell loss, IOP spikes, and high failure rate.

Keywords: CyPass ${ }^{\circledR}$, Glaucoma, Intraocular pressure, Suprachoroidal.

Journal of Current Glaucoma Practice (2019): 10.5005/jp-journals-10078-1264
\end{abstract}

\section{INTRODUCTION}

Glaucoma is the second leading reason for blindness in England and Wales following exudative macular degeneration. ${ }^{1}$ Reduction in intraocular pressure (IOP) is the only modifiable risk factor that may prevent deterioration and visual disability. ${ }^{2}$ Glaucoma treatment has traditionally been initiated with IOPlowering medication (mainly topical treatment) as well as laser treatment. Filtration surgeries have traditionally been reserved for rapidly progressive patients, patients with uncontrolled IOP under maximal tolerable treatment, and those with advanced glaucoma at presentation. Compliance for topical treatment is known to be a barrier to effective IOP reduction with some reports suggesting $<25 \%$ of patients persistent with their topical treatment over 12 months. $^{3}$

A growing body of evidence suggests that minimally invasive glaucoma surgery (MIGS) may change this classic treatment paradigm. The MIGS procedures provide a potentially safe, effective, and simple solution for the mild and moderate progressing glaucoma patient. ${ }^{4}$ A number of novel techniques have been proposed utilizing a range of potential aqueous humor drainage pathways. Devices such as the iStent (Glaukos Corporation, Laguna Hills, CA, USA) and the Hydrus (Ivantis Inc., Irvine, CA, USA) bypass the trabecular meshwork by insertion into the canal of Schlemm. The XEN45 gel implant (Allergan Inc., Irvine, CA, USA) bypasses the trabecular meshwork to drain fluid to the subconjunctival space as in traditional filtration surgery. CyPass ${ }^{\circledR}$ Micro-Stent (Alcon, USA) and the iStent Supra (Glaukos Corporation, Laguna Hills, CA, USA) are injected into the suprachoroidal space to encourage aqueous drainage via this route. Studies of the efficacy of methods utilizing the suprachoroidal space and canal of Schlemm have shown pressure reductions in the mid- to high teens as well as reduced the drop use
${ }^{1}$ Department of Ophthalmology, Rabin Medical Center, Petah Tikva, Israel; Sackler School of Medicine, Tel-Aviv University, Tel-Aviv, Israel

${ }^{2}$ Department of Ophthalmology, Southend University Hospital NHS Foundation Trust, Southend-on-Sea, UK

Corresponding Author: Itay E Gabbay, Department of Ophthalmology, Rabin Medical Center, Petah Tikva, Israel; Sackler School of Medicine, Tel-Aviv University, Tel-Aviv, Israel, Phone: +972 39376101, e-mail: igabbay@gmail.com

How to cite this article: Gabbay IE, Ruben S. CyPass ${ }^{\circledR}$ Micro-Stent Safety and Efficacy at One Year: What Have We Learned? J Curr Glaucoma Pract 2019;13(3):99-103.

Source of support: Nil

Conflict of interest: None

and a favorable safety profile, ${ }^{5-9}$ this comes into question with the recent recall of the CyPass ${ }^{\circledR}$ Micro-Stent (AIcon, USA). The MIGS that use the suprachoroidal pathway have an added advantage that they leave the subconjunctival space untouched for future filtration procedures. The CyPass ${ }^{\circledast}$ Micro-Stent is a fenestrated polyimide stent designed to be inserted into the supraciliary space and facilitates uveoscleral aqueous outflow and lower IOP with minimal complications. $^{10}$

Nonrandomized studies demonstrated significant IOP lowering both as a stand-alone procedure and as combined phacoemulsification, and the CyPass ${ }^{\circledR}$ procedure with no significant adverse events noted, showing an IOP reduction of about $30 \% .^{10-12}$ The COMPASS study was a randomized controlled trial evaluating the 2-year safety and efficacy of supraciliary microstenting, this study showed a sustained reduction in IOP after 2 years of follow-up for mild-to-moderate primary open-angle glaucoma (POAG) patients with no significant side effects. ${ }^{8}$ 
Until trabeculectomy was described in the 1970s, cyclodialysis was a surgical approach to induce hypotension, where the main focus was ways to effectively induce a lasting fistula between the anterior chamber (AC) and the suprachoroidal space. ${ }^{13}$ The technique for cyclodialysis formation changed through the years since the original cyclodialysis technique was described by Heine. ${ }^{14-16}$ In recent years, ab externo cyclodialysis has largely gone out of favor, and some authors describe performing a modified procedure as an adjunct for trabeculectomy. ${ }^{17,18}$ Traumatic cyclodialysis is a rare condition, usually seen following blunt trauma causing severe hypotension through direct communication between the AC and the suprachoroidal space, bypassing the physiological resistance to outflow. ${ }^{19,20}$ Reduced blood supply to the ciliary body may further reduce IOP through reduced production of aqueous humor. ${ }^{21}$ Closure of the ciliary cleft can be accompanied by an IOP spike, which is thought to be caused by a reduction in outflow through the dialysis/bypass with incomplete trabecular meshwork function.

Ab externo suprachoroidal devices have also been described and include the Gold Shunt (SOLX Inc., Waltham, Massachusetts, USA) and STARflo (iSTAR Medical, Isnes, Belgium). These devices require a peritomy and scleral flap dissection and $a b$ externo introduction of the device into the $\mathrm{AC}$, followed by the insertion of the posterior aspect of the shunt into the suprachoroidal space. Published results for the ab externo Gold Shunt approach to the suprachoroidal space show IOP lowering in mid-teens with variable success rate yet large cohort, prospective, long-term results are lacking. ${ }^{22-24}$

Recently, the CyPass ${ }^{\circledR}$ Micro-Stent has been recalled off the market due to concerns raised for endothelial cell loss after 5 years of follow-up (COMPASS XT trial, an extensions of the original COMPASS trial). ${ }^{8}$ While the device is no longer available for use, we believe our data may offer insight for the future use of the suprachoroidal space in clinical practice. We present our experience with the CyPass ${ }^{\circledR}$ Micro-Stent for a heterogeneous cohort of patients in the NHS setting at a university-affiliated regional hospital in the UK.

\section{Materials and Methods}

We present a retrospective (historical prospective), noncomparative audit of patient records that underwent CyPass ${ }^{\circledR}$ Micro-Stent implantation at Southend University Hospital NHS Foundation Trust between June 2017 and August 2018. The audit was approved by the governance unit of Southend University Hospital NHS Foundation Trust, and the tenets of the declaration of Helsinki were strictly upheld. Patients were referred to surgery from the glaucoma clinic after a thorough clinical assessment per our clinical practice. The preoperative examination included medical history, visual acuity testing with a Snellen's chart, slit-lamp examination, IOP measurement (Goldman applanation tonometer), gonioscopic assessment of the AC angle, lens status, and dilated fundoscopy. The optic nerve was assessed clinically and optical coherence tomography was performed as was deemed required by the treating physician. Visual fields were assessed using the Humphrey Visual Field Analyzer (Carl Zeiss AG, Oberkochen, Germany). If both eyes underwent the procedure, each eye was considered as a separate case for analysis.

Patients were referred for surgery based on the following indications:

- Mild-to-moderate POAG with uncontrolled IOP with maximal tolerable medical therapy (MTMT).
- Mild-to-moderate POAG with controlled IOP but visually significant medication side effects, ocular surface disease, or difficulty with topical treatment.

- Patients following failed shunt procedures (i.e., refractory glaucoma patients). These patients had previously undergone trabeculectomy, tube shunt, or XEN45 procedures and have poor pressure control with MTMT.

- Primary angle closure glaucoma (PACG) patients with uncontrolled IOP with MTMT and are pseudophakic or require cataract surgery (this is to allow access to the AC angle).

Per our protocol, all glaucoma medications were discontinued on the day of surgery.

During implantation, the $\mathrm{AC}$ was maintained using viscoelastic (Healon GV; Johnson and Johnson, NJ, USA). CyPass ${ }^{\circledR}$ Micro-Stent was inserted $a b$ interno into position under gonioscopic guidance. In combined cases, phacoemulsification and injection of an intraocular lens was performed first. After removal of the viscoelastic used for lens implantation, miosis was induced using intracameral acetylcholine, followed by reintroduction of viscoelastic agent and $\mathrm{CyPass}^{\circledR}$ implantation. Following the procedure, patients were treated with combined topical steroid and antibiotic (tobramycin $0.3 \%$ and dexamethasone $0.1 \%$ ) four times a day for 3 weeks. Patients were followed up in the glaucoma clinic, and medical treatment or further intervention was added as deemed necessary by the treating physician. Patients were seen in an initial postoperative visit 1 week post-CyPass ${ }^{\circledR}$ implantation. We then collected data from their 1-, 3-, 6-, and 12-month review.

The main outcome measures were IOP reduction and the number of glaucoma drops taken at each time point. Failure was defined as those deemed by the assessing physician to have insufficiently controlled IOP and those who require further glaucoma surgery. Pressure spikes, defined as IOP above $29 \mathrm{~mm} \mathrm{Hg}$, were recorded for each patient. Postoperative IOP measurements and number of drops taken were compared to preoperative values and were analyzed for statistical significance using a paired $t$ test. Failure was examined using Kaplan-Meier survival analysis. All variables are presented as mean \pm standard deviation, $p$ value $<0.05$ was considered statistically significant. Best corrected visual acuity is presented in LogMAR. Statistical analysis was performed with SPSS ${ }^{\circledast} 21$ (IBM, Armonk, NY, USA).

\section{Results}

A total of 77 consecutive case records of 69 patients who underwent the CyPass ${ }^{\circledR}$ procedure between August 2017 and August 2018 were included in the study. Of these, two records were unavailable for analysis and one was excluded as the surgery was cancelled due to uncooperative patient that had later undergone the procedure under general anesthesia. Two patients had died before completing 6 months of follow-up, eight had been lost to follow-up, with no 5-month data available. Sixty-four cases had been analyzed. Cohort baseline characteristics are presented in Table 1. CyPass ${ }^{\circledR}$ was performed as a stand-alone procedure in 37 (58\%) cases, of those 23 (36\% of 64 ) were psudophakic at the time of surgery and 14 (22\% of 64$)$ underwent phakic CyPass ${ }^{\circledR}$ procedure as no significant cataract was present. Combined phacoemulsification and CyPass ${ }^{\circledR}$ procedures were performed in 27 (42\%) cases. Primary open-angle glaucoma was the primary diagnosis in $45(70.3 \%)$ cases, angle closure glaucoma in $3(4.7 \%)$, ocular hypertension in 4 (6.3\%), pigmentary glaucoma in $1(1.6 \%)$, and refractory glaucoma in 11 (17.2\%) cases. 
Of the refractory glaucoma patients, all had undergone prior glaucoma procedures (trabeculectomy, deep sclerectomy, or XEN). The mean follow-up was $10.6 \pm 3.4$ months $(\min .=5.0$ max. $=19.1$ months).

The mean IOP for each time point of follow-up is presented in Table 2. Each postoperative time point showed a statistically significant drop in IOP compared to the preoperative values.

Table 1: Cohort baseline characteristics

\begin{tabular}{lll}
\hline Variable & Category & Summary \\
\hline Age & - & $75.39 \pm 8.58$ \\
Gender & Female & $38(59.4 \%)$ \\
Operated eye & Male & $26(40.6 \%)$ \\
Procedure & Left & $37(57.8 \%)$ \\
& Right & $27(42.2 \%)$ \\
& Stand-alone CyPass ${ }^{\circledR}$ & $37(57.8 \%)$ \\
Primary diagnosis & $14(21.9 \%)$ \\
& Phakic CyPass ${ }^{\circledR}$ & $23(35.9 \%)$ \\
& Pseudophakic CyPass ${ }^{\circledR}$ & $27(42.2 \%)$ \\
& Combined phaco + CyPass ${ }^{\circledR}$ & $45(70.3 \%)$ \\
& POAG & $3(4.7 \%)$ \\
& OHT & $4(6.3 \%)$ \\
& Pigmentary glaucoma & $1(1.6 \%)$ \\
& Refractory glaucoma & $11(17.2 \%)$ \\
\hline
\end{tabular}

Table 2: Main outcome measures-mean intraocular pressure and antihypertensive drop use at each postoperative time point

\begin{tabular}{lllc}
\hline & & $\begin{array}{l}\text { IOP }(\mathrm{mm} \mathrm{Hg}) \\
\text { mean } \pm \text { SD }\end{array}$ & $\begin{array}{c}\text { Drop use (\#) } \\
\text { mean } \pm S D\end{array}$ \\
\hline Preoperative IOP & 64 & $24.63 \pm 7.2$ & $2.41 \pm 0.9$ \\
IOP, 3 months & 55 & $16.24 \pm 7.5$ & $0.31 \pm 0.7$ \\
IOP, 6 months & 44 & $18.39 \pm 10.4$ & $0.52 \pm 1.0$ \\
IOP, 9 months & 28 & $18.18 \pm 8.0$ & $0.86 \pm 1.2$ \\
IOP, 12 months & 27 & $16.41 \pm 4.5$ & $1.11 \pm 1.3$ \\
\hline
\end{tabular}

For all time points $p<0.001$ compared to preoperative values

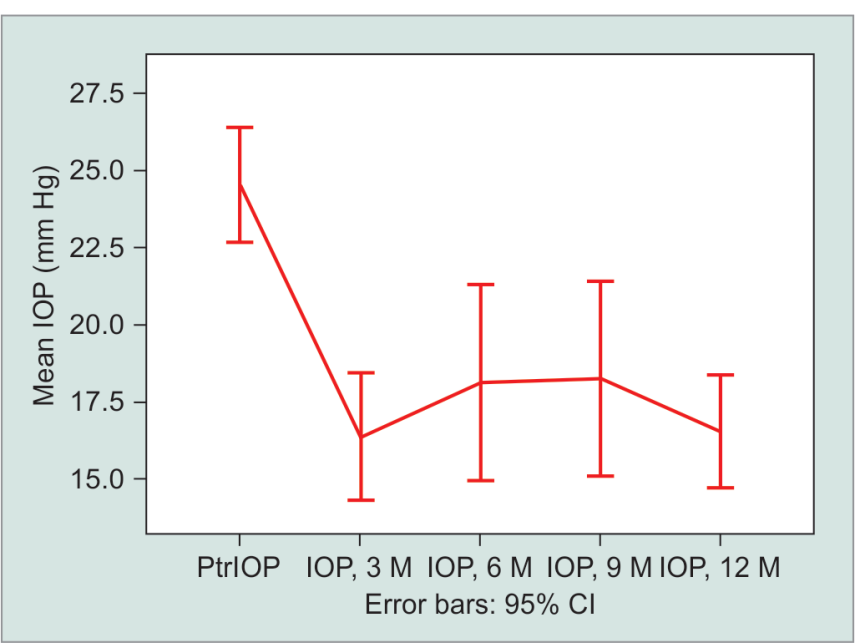

Fig. 1: Mean intraocular pressure preoperatively and at each postoperative time point. Each postoperative time point shows a statistically significant drop in intraocular pressure compared to the preoperative values (Ptr, pretreatment)
At baseline, the mean IOP was $24.6 \pm 7.2 \mathrm{~mm} \mathrm{Hg}$. The mean 3-month postoperative IOP was $16.2 \pm 7.5 \mathrm{~mm} \mathrm{Hg}(p<0.001)$, representing a $34.1 \%$ drop in IOP. The mean IOP at 12-month follow-up was $16.4 \pm$ $4.5 \mathrm{~mm} \mathrm{Hg}(p<0.001)$ representing a 33.3\% drop in IOP. The IOP preoperatively and for each time point in the study are presented in Figure 1. When comparing the stand-alone procedure and combined phaco + CyPass ${ }^{\circledR}$ procedure, the mean IOP at baseline is lower for the combined phaco + CyPass $^{\circledR}$ procedure group $(21.67 \pm 1.0$ vs $26.78 \pm 1.2$ combined vs stand-alone, respectively, $p=0.005)$; and no statistically significant difference was found for the postoperative period, though a trend toward lower IOP in the combined group was noted, which can be seen in Figure 2.

The number of glaucoma medications taken postoperatively was compared to preoperative values and are presented in Table 2. At baseline, the mean number of medications was $2.41 \pm 0.98$. A statistically significant reduction was observed in the mean number of drops taken at 12 months, i.e., to $1.1 \pm 1.3$. At baseline, $60(93.7 \%)$ patients are on one or more antihypertensive drops. At 12 months postoperatively, only 13 (48.1\%) of 27 patients are required to use antihypertensive treatment. Antihypertensive drop use preoperatively and for each time point are presented in Figure 3.

The best corrected visual acuity analysis did not show statistically significant change preoperatively and at each postoperative time point as shown in Figure 4. When comparing the change in visual acuity (VA) stratified by surgery type (combined vs stand-alone procedure), no statistical difference was noted; yet the combined procedure reveals a trend toward an improvement in VA, while the stand-alone procedure remains fairly constant throughout the study period. At the 12-month time point, the VA decreased in the stand-alone group ( $0.12 \pm 0.17$ LogMAR) and improved in the combined group $(-0.19 \pm 0.78 \mathrm{LogMAR})$, yet the difference was not statistically significant $(p=0.135)$.

Survival analysis is presented as a Kaplan-Meier survival estimate graph (Fig. 5). At the 12-month postoperative time point, $24 \%$ was deemed as failure $(76 \%$ of patients was deemed to be free from failure std. error 6\%). Of these, two required cycloablative surgery, two required a trabeculectomy, three

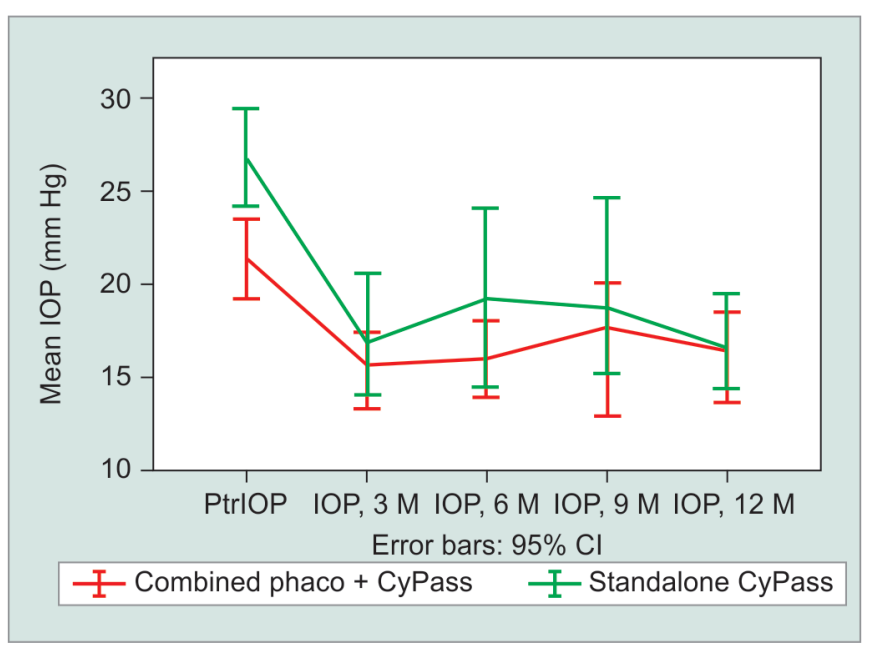

Fig. 2: Mean intraocular pressure preoperatively and at each postoperative time point stratified by procedure type (stand-alone CyPass ${ }^{\circledR}$ vs combined phaco + CyPass $^{\circledR}$ ). Mean intraocular pressure at baseline is lower for the combined phaco + CyPass ${ }^{\circledR}$ vs the stand-alone procedure group. No statistically significant difference was found for the postoperative period, though a trend toward lower intraocular pressure was noted in the combined group 


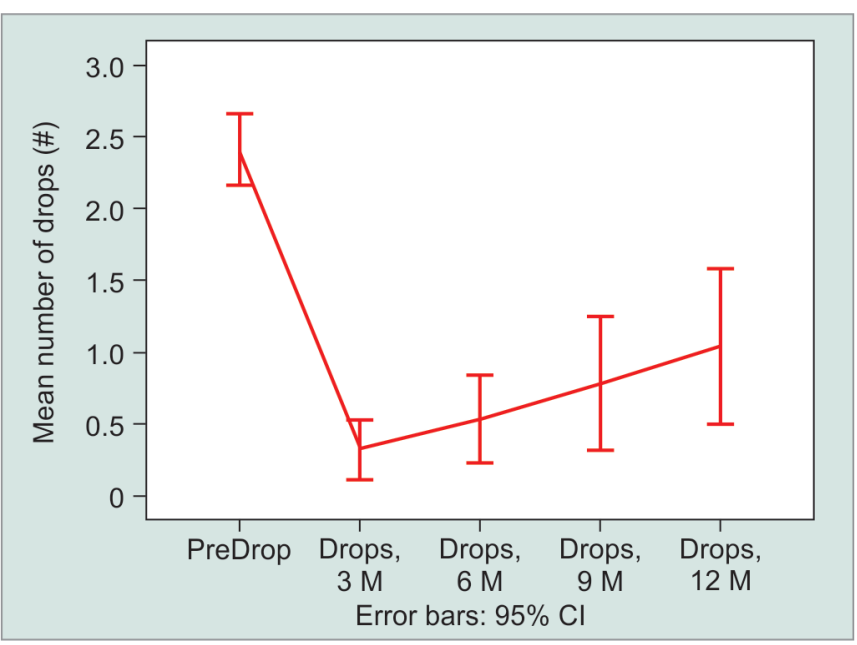

Fig. 3: Mean antihypertensive drop use preoperatively and at each follow-up time point. Each postoperative time point shows a statistically significant reduction in antihypertensive drop use compared to preoperative values

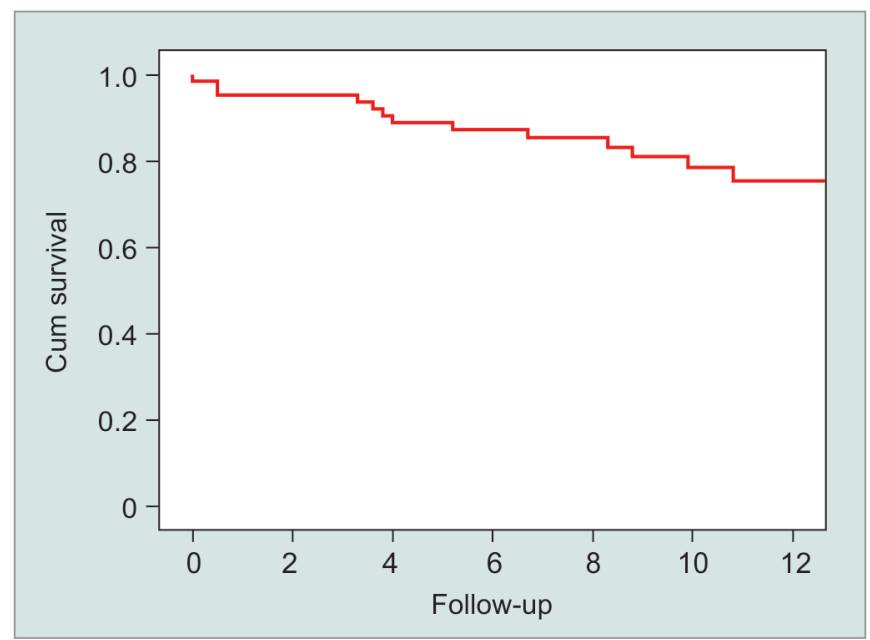

Fig. 5: Kaplan-Meier survival estimate graph. At the 12-month postoperative time point, $24 \%$ were deemed as failure $(76 \%$ of patients were deemed to be free from failure std error $6 \%$ )

required a glaucoma filtration device, four were referred for a XEN45 procedure, one had a dislocated $\mathrm{CyPass}^{\circledR}$ that was removed from the $\mathrm{AC}$, and one had a complete retinal detachment 6 months following the procedure that required vitrectomy with subsequent increase in pressure that required further surgical treatment. Of the 13 failed eyes, $9(70 \%)$ had failed within 6 months of the procedure.

Adverse events-two cases (3.1\%) of transient hyphema, three cases (4.6\%) of choroidal effusion that were (self-resolving), and one case $(1.6 \%)$ of vitreous hemorrhage, which was likely due to malposition of the CyPass ${ }^{\circledR}$. The CyPass $^{\circledR}$ spontaneously extruded into the AC 2 weeks postoperatively and was subsequently removed. One case (1.6\%) presented with subtotal rhegmatogenous retinal detachment 6 months following the CyPass ${ }^{\circledR}$ procedure. The IOP spikes were noted in 18 (28.1\%) cases. Adverse events are summarized in Table 3 . We have not encountered any clinically significant endothelial cell dysfunction.

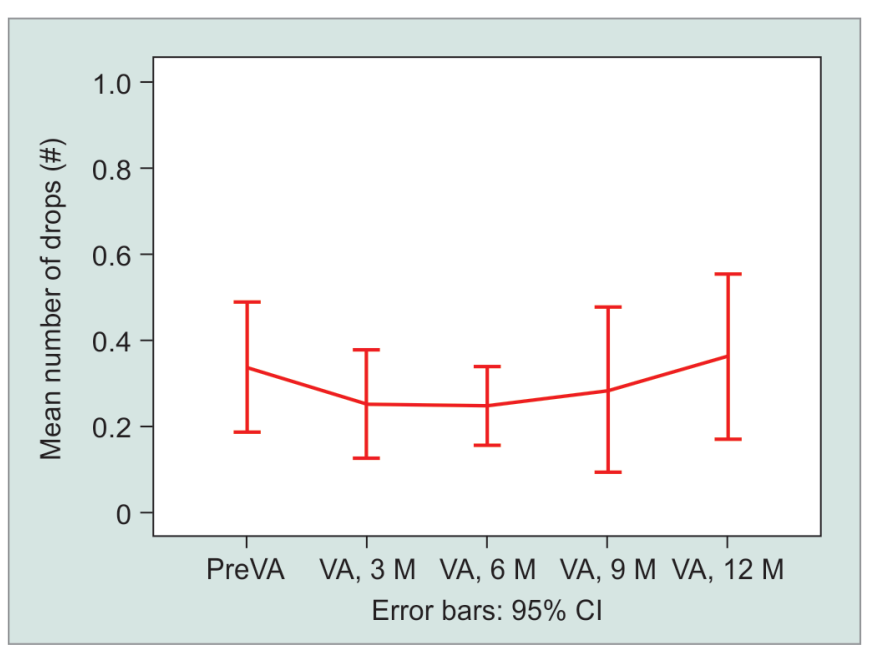

Fig. 4: Mean VA preoperatively and postoperatively at each follow-up time point. The best corrected visual acuity analysis did not show statistically significant change preoperatively and at each postoperative time point when looking at the entire cohort

Table 3: Adverse events

\begin{tabular}{lc}
\hline Adverse events & $n(\%)$ \\
\hline Hyphema & $2(3.1)$ \\
Vitreous hemorrhage & $1(1.6)$ \\
Choroidal effusion & $3(4.7)$ \\
Myopic shift & $1(1.6)$ \\
Retinal detachment & $1(1.6)$ \\
Pressure spikes $(>29 \mathrm{~mm} \mathrm{Hg})$ & $16(28.1)$ \\
\hline
\end{tabular}

\section{Discussion}

We present a retrospective (historical prospective) 1-year clinical audit for a cohort of patients who underwent CyPass ${ }^{\circledR}$ Micro-Stent implementation in a university-affiliated hospital in an NHS setting in the UK. While CyPass ${ }^{\circledR}$ has been pulled off the market amid concerns of endothelial cell damage, we have not encountered any clinically significant endothelial cell dysfunction throughout the study period, yet this appears to be a later event and would require further follow-up. Our study provides insight into the suprachoroidal pathway.

Our data shows a $30 \%$ reduction in the mean IOP at the 12-month time point, with a mean of 1.1 antihypertensive drops (representing a $53 \%$ reduction in drop use). While about $50 \%$ of patients can remain off antihypertensive drops after 12 months. Our data show similar results published in both nonrandomized and randomized studies, demonstrating a mean reduction in IOP of around $30 \% .8,10,11,24,25$ When we compare stand-alone vs combined phaco $+\mathrm{CyPass}^{\circledR}$ procedures, lower baseline IOP is observed in the combined group, which may represent a lower threshold for referral for patients who require cataract surgery and can be offered CyPass ${ }^{\circledR}$ as an add-on procedure.

Over the study period, $30 \%$ of cases present with IOP spikes over $29 \mathrm{~mm} \mathrm{Hg}$, and most have responded well to topical medication. The IOP spikes present as an acute IOP rise with no abnormality on examination-the AC is deep and quiet, and the CyPass ${ }^{\circledR}$ microshunt can be seen through the gonio lens with no apparent block to its proximal side. The main concern with these pressure spikes is that 
some may go unnoticed as they do not generally cause discomfort, pain, or blurred vision but may leave patients with increased pressure over long periods of time between follow-up visits.

We hypothesize the IOP spikes seen with the use of the CyPass ${ }^{\circledR}$ micro-shunt may follow a similar pattern to AC cleft closure. As long as the outflow to the suprachoroidal space through (or around) the CyPass ${ }^{\circledR}$ is patent, pressure remains low. Flow obstruction may be caused by obstruction in the device itself or by scarring in the suprachoroidal space. Once the bypass has been blocked, reduced trabecular meshwork function causes the IOP to abruptly rise.

Study limitations - our results are limited by their retrospective nature yet may offer insight into real-world results. Patients using public health systems as the NHS miss their appointments and may be lost to follow-up. This presents a clinical challenge and could create bias toward patients who are more involved in their treatment. In both pseudophakic and combined procedure patients, some IOP reduction related to cataract extraction may be included. Inclusion of several glaucoma types may further bias the results as one subgroup may disproportionally contribute to the mean IOP (the number of patients in non-POAG groups were small, and this did not allow for a significant subgroup analysis). One could expect that patients with prior failed surgery may be at high risk, yet subgroup analysis for this small group of patients did not yield statistically different results compared to patients who did not have prior glaucoma surgery. Prospective, randomized, controlled studies are required to better establish efficacy and safety for each disease subtype and glaucoma severity level and to correlate pressure reduction with visual field progression.

In conclusion, the suprachoroidal pathway appears to potentially offer a good alternative to the classic subconjunctival pathways, yet presents a new set of challenges not previously encountered through the more classic subconjunctival pathway. Further studies and future minimally invasive procedures may harness the suprachoroidal pathway to offer patients IOP reduction but would have to address the concerns such as endothelial cell loss, IOP spikes, and high failure rate.

\section{References}

1. Bunce C, Wormald R. Causes of blind certifications in England and Wales: April 1999-March 2000. Eye (Lond) 2008;22(7):905-911. DOI: 10.1038/sj.eye.6702767.

2. Heijl A, Leske MC, Bengtsson B, et al. Reduction of intraocular pressure and glaucoma progression: results from the early manifest glaucoma trial. Arch Ophthalmol 2002;120(10):1268-1279. DOI: 10.1001/archopht.120.10.1268.

3. Schwartz GF. Compliance and persistency in glaucoma follow-up treatment. Curr Opin Ophthalmol 2005;16(2):114-121. DOI: 10.1097/01. icu.0000156139.05323.26.

4. Kerr NM, Wang J, Barton K. Minimally invasive glaucoma surgery as primary stand-alone surgery for glaucoma. Clin Exp Ophthalmol 2017;45(4):393-400. DOI: 10.1111/ceo.12888.

5. Hoeh H, Vold SD, Ahmed IK, et al. Initial clinical experience with the CyPass Micro-Stent: safety and surgical outcomes of a novel supraciliary microstent. J Glaucoma 2016;25(1):106-112. DOI: 10.1097/ IJG.0000000000000134.

6. Pfeiffer N, Garcia-Feijoo J, Martinez-de-la-Casa JM, et al. A randomized trial of a Schlemm's canal Microstent with phacoemulsification for reducing intraocular pressure in open-angle glaucoma. Ophthalmology 2015;122(7):1283-1293. DOI: 10.1016/j.ophtha.2015.03.031.
7. Arriola-Villalobos $P$, Martínez-de-la-Casa JM, Díaz-Valle $D$, et al. Combined iStent trabecular micro-bypass stent implantation and phacoemulsification for coexistent open-angle glaucoma and cataract: a long-term study. Br J Ophthalmol 2012;96(5):645-649. DOI: 10.1136/bjophthalmol-2011-300218.

8. Vold S, Ahmed IIK, Craven ER, et al. Two-year COMPASS trial results: supraciliary Microstenting with phacoemulsification in patients with open-angle glaucoma and cataracts. Ophthalmology 2016;123(10):2103-2112. DOI: 10.1016/j.ophtha.2016.06.032.

9. Craven ER, Katz LJ, Wells JM, et al. Cataract surgery with trabecular micro-bypass stent implantation in patients with mild-to-moderate open-angle glaucoma and cataract: two-year follow-up. J Cataract Refract Surg 2012;38(8):1339-1345. DOI: 10.1016/j.jcrs.2012.03.025.

10. Hoeh H, Ahmed IIK, Grisanti S, et al. Early postoperative safety and surgical outcomes after implantation of a suprachoroidal Micro-Stent for the treatment of open-angle glaucoma concomitant with cataract surgery. J Cataract Refract Surg 2013;39(3):431-437. DOI: 10.1016/ j.jcrs.2012.10.040.

11. García-Feijoo J, Rau M, Grisanti S, et al. Supraciliary Micro-Stent implantation for open-angle glaucoma failing topical therapy: 1-year results of a Multicenter study. Am J Ophthalmol 2015;159(6): 1075.e1-1081.e1. DOI: 10.1016/j.ajo.2015.02.018.

12. Höh H, Grisanti S, Grisanti S, et al. Two-year clinical experience with the CyPass Micro-Stent: safety and surgical outcomes of a novel supraciliary Micro-Stent. Klin Monatsbl Augenheilkd 2014;231(4): 377-381. DOI: 10.1055/s-0034-1368214.

13. González-Martín-Moro J, Contreras-Martín I, Muñoz-Negrete FJ, et al. Cyclodialysis: an update. Int Ophthalmol 2017;37(2):441-457. DOI: 10.1007/s10792-016-0282-8.

14. Barkan O, Boyle SF, Maisler S. On the surgery of glaucoma: mode of action of cyclodialysis. Calif West Med 1936;44(1):12-16. DOI: 10.1016/ S0002-9394(36)95189-9.

15. Sédan J. The present development of cyclodialysis from 1905 to 1967. Ann Ocul (Paris) 1967;200(3):293-306.

16. Barkan O. Cyclodialysis, multiple or single, with air injection-an operative technique for chronic glaucoma. Calif Med 1947;67(2): 78-83. DOI: 10.1016/0002-9394(47)91090-8.

17. Dada T, Sharma R, Sinha G, et al. Cyclodialysis-wnhanced trabeculectomy with triple Ologen implantation. Eur J Ophthalmol 2016;26(1):95-97. DOI: 10.5301/ejo.5000633.

18. Skalicky SE, Lew HR. Surgical outcomes of combined trabeculectomycyclodialysis for glaucoma. J Glaucoma 2015;24(1):37-44. DOI: 10.1097/JJG.0b013e3182883c44.

19. Brubaker RF, Pederson JE. Ciliochoroidal detachment. Surv Ophthalmol 1983;27(5):281-289. DOI: 10.1016/0039-6257(83)90228-X.

20. Ormerod LD, Baerveldt G, Sunalp MA, et al. Management of the hypotonous cyclodialysis cleft. Ophthalmology 1991;98(9):1384-1393. DOI: 10.1016/S0161-6420(91)32121-3.

21. Malandrini A, Balestrazzi A, Martone G, et al. Diagnosis and management of traumatic cyclodialysis cleft. J Cataract Refract Surg 2008;34(7):1213-1216. DOI: 10.1016/j.jcrs.2008.02.038.

22. Melamed S, Ben Simon GJ, Goldenfeld M, et al. Efficacy and safety of gold micro shunt implantation to the supraciliary space in patients with glaucoma: a pilot study. Arch Ophthalmol 2009;127(3):264-269. DOI: 10.1001/archophthalmol.2008.611.

23. Cseke I, Vámosi P, Bausz M. Starflo glaucoma implant: early experience in Hungary. Rom J Ophthalmol 2016;60(1):14-17.

24. Figus $M$, Lazzeri $S$, Fogagnolo $P$, et al. Supraciliary shunt in refractory glaucoma. Br J Ophthalmol 2011;95(11):1537-1541. DOI: 10.1136/ bjophthalmol-2011-300308.

25. Fili S, Seddig S, Papadopoulou E, et al. 1-Jahres-Ergebnisse nach Cypass-implantation [one-year results after Cypass implantation]. Ophthalmologe 2019;116(5):447-455. DOI: 10.1007/s00347-0180745-6. 